quantitative data. This was distributed via email to all doctors at CRH in September 2018 three months after the implementation date. Results were analysed and distributed to stakeholders.

Results A total of 43 doctors completed the survey, representing a range of grades and specialties. Mean score for how user-friendly respondents found the form was 6 out of 10 . The proportion of respondents who found ReSPECT useful in planning patient care was 58\%. However, approximately three quarters of respondents had encountered difficulties with the form $(74 \%)$.

Six broad themes around difficulties with ReSPECT were identified: 1) Time required, 2) CPR decision, 3) Complexity, 4) Scale - life sustaining vs comfort, 5) Paper format and storage issues and 6) Capacity assessment. Suggestions for improvement of the ReSPECT process included: larger boxes on the form, clearer emphasis on CPR decision, and improving systems for handover between care settings.

Conclusion The ReSPECT process has implications for the palliative care of patients admitted to hospital. The results of this survey demonstrate concerns from clinicians, and suggestions for improvement. The suggestions of CRH staff could provide a basis for guidance for other implementers and improvements to future versions of the form nationally.

\section{REDESIGNING SPECIALIST PALLIATIVE AND HOSPICE CARE TO MEET THE NEEDS OF PEOPLE WITH FRAILTY}

Caroline Nicholson, Rob George, Victor Pace, Heather Richardson. St Christopher's Hospice, King's College London

\subsection{6/bmjspcare-2019-ASP.114}

Background Older people with multimorbidities will dominate palliative care in coming decades. For them, dying can be slow and unpredictable. Care models integrated across the spectrum of geriatric and palliative medicine, health and social care, are emerging. We draw on current theory and evidence to develop a framework that assists current services attune to the needs of this population.

Methods We used stepped framework construction.Eight clinicians and service users reviewed and refined initial findings.

- to scope the palliative/geriatric literature and define focus, inclusion and exclusion criteria for a proactive palliative care model (Seymour 2014). Documents from 102 research, review, policy papers and grey literature were included.

- Face-validity testing extended to a wider consultation across care sectors, care commissioners and providers.

Findings Built around emergent concerns of recipient older people and their family as care partners, the framework identifies three intervention levels that work

- differently as clinicians;

- in partnership to improve services and the broader care system;

- $\quad$ as support to wider societal change.

An optimal clinical response requires:

- Meticulous, systematic needs assessment using the right tools;

- Parallel planning across specialties for several possible outcomes;
- Sensitive discussions that are realistic about health conditions and age;

- Access to advanced palliative practice competent to stop as well as start treatments, and work across relevant specialties including rapid response in the community;

- Access to rehabilitative care that optimizes function;

- 'Watchful Waiting', where purposeful, low-level proactive engagement with people and families identifies incremental changes and responds appropriately;

- Effective, relevant bereavement care.

Outcome This Age Attuned Hospice framework offers a flexible structure for organisations seeking to become more responsive to the older, frail population whilst maintaining effective current specialist practice.

\section{INTRODUCING THE TRAJECTORY TOUCHPOINT TECHNIQUE: A SYSTEMATIC METHODOLOGY FOR CAPTURING THE VOICES OF PALLIATIVE CARE SERVICE USERS}

Lynn Sudbury-Riley, Philippa Hunter-Jones, Ahmed Al-Abdin. University of Liverpool

\subsection{6/bmjspcare-2019-ASP.115}

Background We present the trajectory touchpoint technique (TTT), a new methodology to appreciate all user experiences of palliative care. Better understanding of user needs means providers can become more patient-centered. However, no available tools capture experience of all aspects of palliative care. Consequently we developed the new methodology, allowing a deep dive into the palliative care journeys of patients and their families.

Methods Using concepts from the social sciences, we blended service blueprinting, customer journeys, touchpoints, servicescapes, experienced based co-design, and rich pictures methodology; producing a technique that systematically captures palliative care user experiences from seven major dimensions. The technique incorporates all aspects of palliative care that are important to the user, integrating emotional, physical, sensorial, and social elements of their experience. We have tested and refined the TTT in a collaboration with hospices and palliative care providers $(n=8)$, successfully capturing the voices $(n=223)$ of palliative care patients and their families.

Results The TTT overcomes many data collection difficulties typical to palliative care. The TTT is easy to understand and use, yet costs a fraction of alternative techniques while allowing for a broader perspective into patient-centeredness, incorporating both the clinical encounter and macro-level organizational structures and processes. Importantly, results demonstrate the TTT's ability to uncover areas for improvement that alternative methodologies have failed to identify, despite many of our collaborating organizations already being rated as 'outstanding'.

Conclusions Data collection methods that reveal actionable results which lead to meaningful changes are crucial to implementing improvements to palliative care. Our TTT responds to this need. Based on our results, our collaborating organizations have already begun to make improvements to the ways in which palliative care services are designed, delivered, communicated, co-created, and connected to the wider health care ecosystem. 